\title{
Right lower lobectomy after right upper lobectomy for multiple metastases in lung cancer of the right lower lobe: Benefit of middle lobe preservation
}

\author{
Hisashi Iwata, MD, PhD, a Takuji Kiryu, MD, PhD, ${ }^{\text {b }}$ Koyo Shirahashi, MD, ${ }^{a}$ \\ Shinsuke Matsumoto, MD, ${ }^{a}$ Masafumi Matsui, MD, and

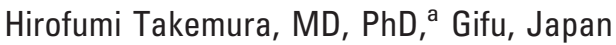

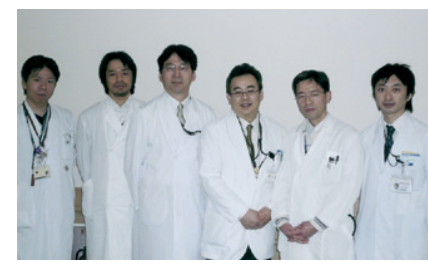

Drs Matsumoto, Shirahashi, Takemura, Iwata, Kiryu, and Matsui (left to right)
$\mathrm{P}$ ostoperative respiratory function is a crucial factor when considering surgical treatment for lung cancer, particularly for relapse or secondary lung cancer. We describe herein a case of lobectomy for multiple metastases in the right lower lobe of the lung with preservation of the middle lobe after initial right upper lobectomy.

\section{Clinical Summary}

A 60-year-old woman with lung cancer underwent right upper lobectomy. The tumor specimen was a well-differentiated adenocarcinoma, pathologic stage IA (T1 N0 M0). Multiple slow-growing nodules were noted in the right lower lobe 4 years postoperatively. Recurrent lesions are generally multiple and disseminated, and additional surgical intervention is not usually indicated. However, no nodules were apparent in other lobes during 1 year of follow-up. The patient was therefore referred to our department for surgical treatment. Routine blood biochemistry and coagulation studies yielded normal results and carcinoembryonic antigen level was 2.6 $\mathrm{ng} / \mathrm{mL}$. A chest computed tomographic scan from 4 years earlier showed the primary lung cancer as a solid tumor in the right upper lobe (Figure 1, A), compared with the scan of multiple nodules limited to the right lower lobe for the metastases (Figure 1, B). These multiple tumors showed no uptake on a fluorodeoxyglucose positron emission tomographic scan. Transbronchiolar lung biopsy was not performed owing to patient refusal. No extrathoracic metastatic lesions were identified. Preoperative forced vital capacity $(\mathrm{FVC})$ and forced expiratory volume in 1 second $\left(\mathrm{FEV}_{1.0}\right)$ were $2420 \mathrm{~mL}$ and $1510 \mathrm{~mL}$, respectively. We evaluated predicted results of completion pnuemonectomy using perfusion scintigraphy and pulmonary artery obstruction testing. Predicted FVC and $\mathrm{FEV}_{1.0}$ after completion pneumonectomy were $1114 \mathrm{~mL}$ and 695 $\mathrm{mL}$, respectively. Obstruction of the right pulmonary artery

\footnotetext{
From the Departments of General and Cardiothoracic Surgery a and Radiology, ${ }^{\mathrm{b}}$ Graduate School of Medicine, Gifu University, Gifu, Japan.

Received for publication Dec 29, 2006; revisions received Feb 13, 2007; accepted for publication Feb 23, 2007.

Address for reprints: Hisashi Iwata, MD, PhD, Department of General and Cardiothoracic Surgery, Graduate School of Medicine, Gifu University, 1-1 Yanagido, Gifu City, Gifu 5011194, Japan (E-mail: ihisashi@gifu-u.ac.jp).

J Thorac Cardiovasc Surg 2007;134:1078-80

$0022-5223 / \$ 32.00$

Copyright $\odot 2007$ by The American Association for Thoracic Surgery doi:10.1016/j.jtcvs.2007.02.054
}

yielded a pressure of $19 \mathrm{~mm} \mathrm{Hg}$. Video-assisted right lower lobectomy was performed, preserving the middle lobe. Adhesion of the lower lobe to the chest wall was easily removed by the thoracoscopic view. The middle lobe was also adherent to the chest wall and helped to prevent middle lobe torsion. On final pathologic examination, the specimen from the lower lobe was characteristic of metastatic adenocarcinoma from the previous lung cancer of the right upper lobe (Figure 1,C and D). Postoperative FVC and $\mathrm{FEV}_{1.0}$ were $1570 \mathrm{~mL}$ and $1200 \mathrm{~mL}$, respectively. Perfusion scintigraphy showed $391,199(21.7 \%)$ of $1,801,504$ counts for the preserved middle lobe (Figure 2). Respiratory function after right upper and lower lobectomies preserved more than half of the original respiratory function.

The postoperative course was uneventful. Chest computed tomographic imaging after 1 year of preservation of the middle lobe showed adequate volume without emphysematous change. After 17 months of follow-up, the patient is doing well with no evidence of disease or requirement for additional oxygen support.

\section{Discussion}

Martini and Melamed ${ }^{1}$ described the properties of metachronous lung cancer such as second cancer in different lobes or lungs but displaying the same histologic features, with neither carcinoma in lymphatics common to both cancer nor extrapulmonary metastasis at the time of diagnosis. However, they also mentioned that bronchial alveolar carcinoma or multinodular growth patterns were arbitrarily excluded because of difficulties in ruling out airborne or intrapulmonary lymphatic dissemination.

The lower lobe specimen displayed characteristics of metastatic adenocarcinoma, given the distinct margin between tumor and normal lung tissue. In addition, multiple tumors were limited to the right lower lobe, suggesting metastases rather than metachronous tumors. We speculated that recurrence was due to transbronchial metastasis during upper lobectomy. From the perspective of management, differentiating between new primary tumors and recurrence is unimportant. ${ }^{2}$ Angeletti and associates ${ }^{3}$ showed that aggressive surgical approaches are safe, effective, and warranted in patients with second primary lung cancer or relapse from primary lung cancer. They analyzed 19 patients with metachronous second primary cancer and 7 patients with local relapse. However, right upper and lower lobectomies while preserving the middle lobe were not performed. Gugginoa and colleagues ${ }^{4}$ described postoperative respiratory insufficiency after completion pneumonectomy in $26.3 \%$ of patients with lung cancer and also reported operative mortality rates of $0 \%$ to $17.6 \%$ for cancer from several institutions. We therefore decided to avoid completion pneumonectomy. Pres- 
A

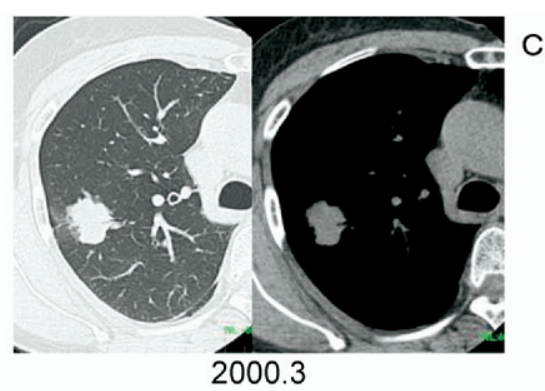

B

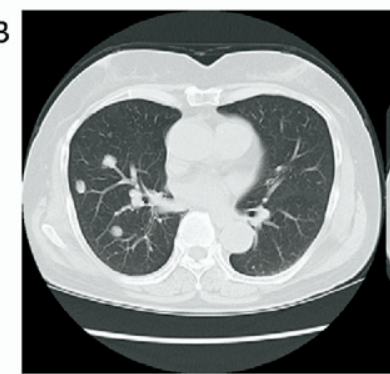

2004.6

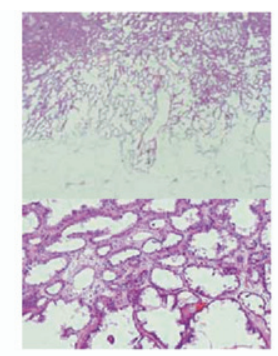

D

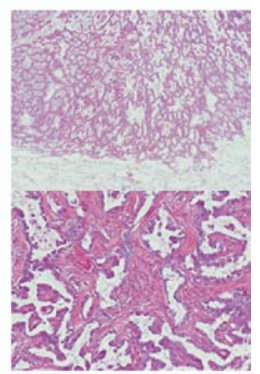

Figure 1. A, Chest computed tomographic scan shows a solid, irregular mass in the right upper lobe at primary operation. B, Chest computed tomographic scan shows multiple, slow-growing masses limited to the right lower lobe. C, Tumor of the right upper lobe, characteristic of well-differentiated adenocarcinoma. D, A specimen from the lower lobe shows clear margin between carcinoma and normal tissue, characteristic of metastatic adenocarcinoma from the primary lesion.

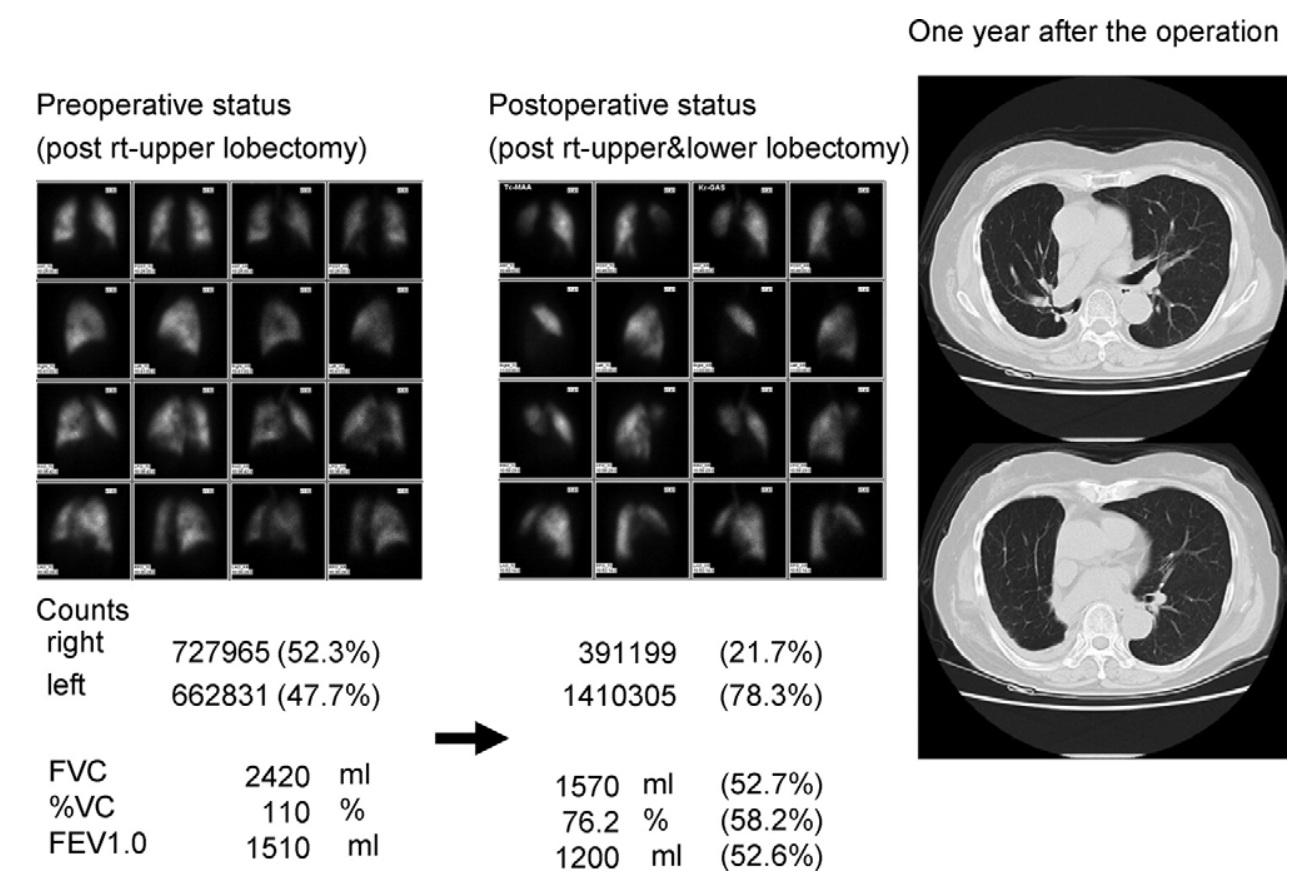

Figure 2. Perfusion scintigraphy of the preserved middle lobe shows a count of 391,199 , indicating $21.7 \%$ residual respiratory function. Preserved forced vital capacity (FVC), percent vital capacity (VC), and forced expiratory volume in 1 second $\left(\mathrm{FEV}_{\mathbf{1 . 0}}\right)$ after right upper and lower lobectomy were at $52.7 \%$, $58.2 \%$ and $\mathbf{5 2 . 5 \%}$ of original respiratory function. The chest computed tomographic image was taken 1 year after preservation of the middle lobe. 
ervation of the middle lobe contributed substantially to postoperative respiratory function (Figure 2).

Conversely, preservation of the middle lobe in the absence of the upper and lower lobes may induce torsion. Various factors appear to be associated with lobar torsion, including complete interlobar fissure, absence of adhesions, a narrow middle lobe hilum, and overzealous mobilization of the lobe. ${ }^{5}$ In our case, the middle lobe was fixed by adhesions to the chest wall. However, emphysematous changes in the residual middle lobe may be induced owing to volume mismatch in the middle lobe and thoracic cavity during long-term follow-up.

In conclusion, preserving the middle lobe after resection of both upper and lower lobes offers advantages to residual respiratory function, compared with results from completion pneumonectomy.

\section{References}

1. Martini N, Melamed MR. Multiple primary lung caners. $J$ Thorac Cardiovascular Surg. 1975;70:606-12.

2. Deschamps C, Palrolero PC, Trastek VF, Payne WS. Multiple primary lung cancers. J Thorac Cardiovasc Surg. 1990;99:769-78.

3. Angeletti CA, Mussi A, Janni A, Lucchi M, Ribechini A, Chella A, et al. Second primary lung cancer and relapse: treatment and follow-up. Eur J Cardiothorac Surg. 1995;9:607-11.

4. Gugginoa G, Doddolia C, Barlesi F, Acri P, Chetaille B, Thomas P, et al. Completion pneumonectomy in cancer patients: experience with 55 cases. Eur J Cardiothorac Surg. 2004;25:449-55.

5. Apostolakis E, Koletsis EN, Panagopoulos N, Prokakis C, Dougenis D. Fatal stroke after completion pneumonectomy for torsion of left upper lobe following left lower lobectomy. J Cardiothorac Surg. 2006; $1: 25$.

\title{
Fibroepithelial polyps: Preoperative diagnosis may avoid thoracotomy
}

\author{
Christopher T. Wartmann, MD, ${ }^{a, b}$ Denise Fernandez, BA, ${ }^{b}$ and Raja M. Flores, MD, ${ }^{b}$ \\ Chicago, III, and New York, NY
}

W e present the case of a 32-year-old man who presented with an indeterminate obstructing lung lesion thought to be malignant. Thoracotomy revealed the lesion to be a benign fibroepithelial polyp of the bronchus. Had this diagnosis been entertained preoperatively, thoracotomy might have been avoided.

\section{Clinical Summary}

This construction worker with a medical history consisting of smoking 1 pack of cigarettes per day for 16 years presented to his general practitioner and was treated for asthma and recurrent pneumonia. A chest radiograph taken at this time showed minimal lower lobe subsegmental atelectatic changes along with a mild degree of perihilar pulmonary vascular congestion. A chest computed tomographic (CT) scan demonstrated a $3-\mathrm{cm}$ mass occluding the left mainstem bronchus, extending into the left upper and lower lobe bronchi (Figure 1). A positron emission CT scan illustrated no

From the Department of Surgery, ${ }^{a}$ Northwestern University, Chicago, Ill; and the Thoracic Service, ${ }^{\mathrm{b}}$ Department of Surgery, Memorial Sloan Kettering Cancer Center, New York, NY.

Received for publication April 23, 2007; accepted for publication May 30, 2007.

Address for reprints: Raja M. Flores, MD, Thoracic Service, Department of Surgery, Memorial Sloan Kettering Cancer Center, 1275 York Ave, New York, NY 10021 (E-mail: floresr@mskcc.org).

J Thorac Cardiovasc Surg 2007;134:1080-1

$0022-5223 / \$ 32.00$

Copyright $\odot 2007$ by The American Association for Thoracic Surgery doi:10.1016/j.jtcvs.2007.05.032 lymphadenopathy, and no fluorodeoxyglucose accumulation was seen in the left endobronchial lesion.

The patient underwent rigid bronchoscopic scanning, where the lesion within the

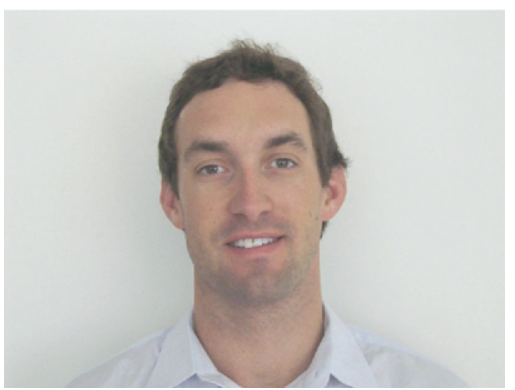

Dr Wartmann left mainstem bronchus was identified. The le-

sion side underwent biopsy multiple times; pathology showed benign squamous mucosa with extensive submucosal fibrosis and mild chronic inflammation, with no tumor seen. An attempt was made at coring out this lesion. However, in lieu of radiographic evaluation and demarcation, it was deemed unsafe to remove at this time because of the potential for pulmonary artery invasion; therefore aggressive coring out by means of rigid bronchoscopy could result in significant hemorrhage. Thus thoracotomy was scheduled because of fear of a missed malignant diagnosis caused by the lesion's extensive nature and to avoid potential hemorrhage caused by performing aggressive rigid bronchoscopy. A left posterolateral thoracotomy was performed, and the lung was inspected without any evidence of parenchymal lesions. A bronchotomy was made in the orifice of the left upper lobe bronchus. A significant amount of endobronchial lesion was removed in a piecemeal fashion. This was a lobulated, multiseptated, fleshy lesion slightly larger than a golf ball that continued down into the left upper and lower lobes (Figure 2). On closer inspection, the lesion was adherent to a segmental bronchus down in the left lower lobe by a single stalk; a frozen section was determined to be benign. The 